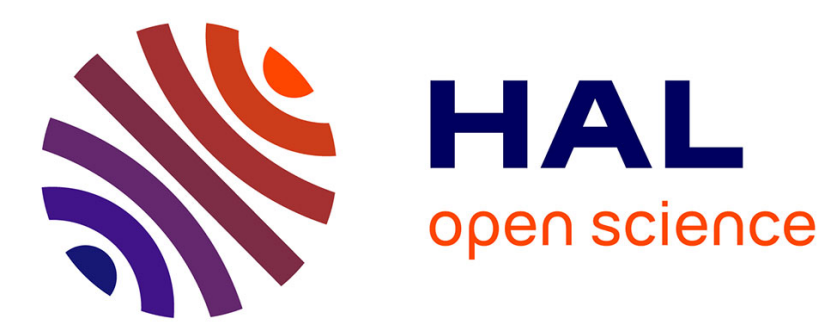

\title{
GÉNÉRATEURS DES POTENTIELS ÉVOQUÉS AUDITIFS CORTICAUX CHEZ L'HOMME
}

\author{
C. Liegeois-Chauvel, A. Musolino, Patrick Chauvel
}

\section{To cite this version:}

C. Liegeois-Chauvel, A. Musolino, Patrick Chauvel. GÉNÉRATEURS DES POTENTIELS ÉVOQUÉS AUDITIFS CORTICAUX CHEZ L'HOMME. Journal de Physique Colloques, 1990, 51 (C2), pp.C2-135-C2-138. 10.1051/jphyscol:1990232 . jpa-00230652

\section{HAL Id: jpa-00230652 https://hal.science/jpa-00230652}

Submitted on 1 Jan 1990

HAL is a multi-disciplinary open access archive for the deposit and dissemination of scientific research documents, whether they are published or not. The documents may come from teaching and research institutions in France or abroad, or from public or private research centers.
L'archive ouverte pluridisciplinaire HAL, est destinée au dépôt et à la diffusion de documents scientifiques de niveau recherche, publiés ou non, émanant des établissements d'enseignement et de recherche français ou étrangers, des laboratoires publics ou privés. 
COLLOQUE DE PHYSIQUE

Colloque C2, supplément au $\mathrm{n}^{\circ} 2$, Tome 51, Février 1990

ler Congrès Français d'Acoustique 1990

GENERATEURS DES POTENTIELS EVOQUES AUDITIFS CORTICAUX CHEZ L HOMME

\author{
C. LIEGEOIS-CHAUVEL, A. MUSOLINO* et P. CHAUVEL \\ INSERM U97, 2 ter, Rue d'Alésia, F-75014 Paris, France \\ * Hôpital Sainte Anne, Service de Neurochirurgie, I Rue Cabanis, \\ F-75014 Paris, France
}

Résumé-Des potentiels évoqués en réponse à des stimulations auditives ont été enregistrés avec des électrodes intracérébrales en différents sites du Gyrus Temporal Transverse (gyrus de Heschl) et du Planum temporale chez des patients épileptiques, candidats à une chirurgie. Les résultats montrent qu'il existe une ségrégation anatomique des réponses évoquées en fonction de leur latence. Les réponses à courte latence $(13-17 \mathrm{msec})$ sont enregistrées préférentiellement dans la région dorso-médiane du gyrus de Heschl, les réponses à moyenne latence $(50-70 \mathrm{msec})$ dans la partie plus latérale et les réponses à longue latence $(>100 \mathrm{msec}$ ) dans le Planum Temporale. Il apparaît que ce sont les réponses à moyenne latence qui codent pour la fréquence tonale.

Abstrat- Auditory evoked potentials have been recorded with depth electrodes in different sites in Temporal Transverse Gyrus (Heschl's gyrus) and in Planum Temporale in epileptic patients that are candidates for neurosurgical corticectomy. Our results show that there exists an anatomical segregation of the evoked response as a function of latency. Short latency responses (13-17 $\mathrm{msec})$ are recorded in the dorso-medial part of the gyrus, middle latency responses (50-70 $\mathrm{msec}$ ) in the more lateral part and long latency responses (>100 $\mathrm{msec}$ ) in the Planum Temporale. Only middle latency evoked potentials vary with the tonal frequency.

\title{
1-INTRODUCTION
}

Les potentiels évoqués auditifs (PEA) enregistrés sur le scalp sont caractérisés par trois groupes de composantes dont le maximum d'amplitude culmine au vertex. L'origine des composantes précoces $(2-9 \mathrm{msec})$ est bien connue et correspond aux différents relais du tronc cérébral . Par contre celle des composantes à moyenne latence $(15-50 \mathrm{msec})$ et à longue latence $(100 \mathrm{msec})$ restent à être clairement identifiées. Des enregistrements intracérébraux sont nécessaires pour localiser les différents générateurs de ces potentiels. Peu d'études ont été faites chez l'Homme. Chatrian et col (1960), puis Celesia et Puletti (1969) ont enregistré des PEA de courte latence (16-30 msec) au niveau du gyrus temporal supérieur mais le nombre limité de sites d'enregistrement ne leur a pas permis de faire une étude extensive du cortex auditif.

Chez des patients épileptiques, candidats à une chirurgie, nous avons enregistré des potentiels évoqués en réponse à différents stimuli auditifs au niveau d'électrodes intracérébrales localisées dans le cortex auditif (gyrus de Heschl et Planum temporale). Une reconstruction anatomique précise de chaque site d'enregistrement a été faite à l'aide de la méthode d'angiographie stéréotaxique développée à l'Hôpital Ste Anne par Szikla et coll (1977).

\section{2-METHODES}

Les enregistrements ont été effectués sur 20 patients (âgés de 16-44 ans ) ayant une épilepsie temporale, rebelle à tout traitement médicamenteux, candidats à une chirurgie.

Afin de localiser la zone épileptogène, chaque patient a une exploration stéréoélectroencéphalographique avec des électrodes profondes. Tous les patients sont informés du déroulement de chaque étape de l'exploration et ont donné leur consentement pour les différents protocoles. Pendant les séances d'enregistrement, ils sont confortablement allongés et restent vigilants . 


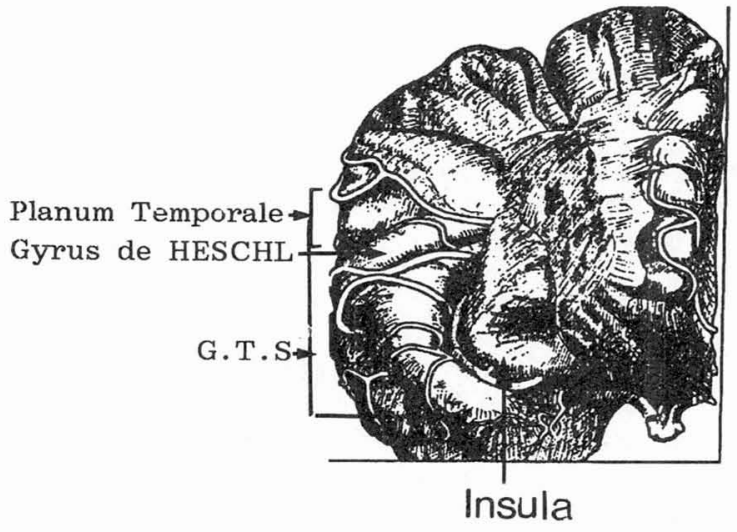

Chaque électrode profonde $(0.8 \mathrm{~mm}$ de diamètre) est constituée de plots étagés (de 5 à 15) équidistants, séparés par des bagues isolantes. La localisation anatomique peut être faite avec une grande précision en utilisant la méthode d'angiographie stéréotaxique développée par Szikla et al (1977), comparée avec les images de résonnance magnétique nucléaire fạite après l'ablation des électrodes (Musolino et al, 1989).

Fig 1 Schéma anatomique de la position du Gyrus de Heschl et du Planum Temporale après dissection $d u$ lobe frontal

La Fig 1 illustre la position du gyrus de Heschl au niveau du gyrus Temporal Supérieur Tous les enregistrements sont monopolaires, chaque plot de l'électrode référencé à un site extracéphalique (collier fermé). Les potentiels évoqués intracérébraux sont simultanément amplifiés, filtrés $(.5 \mathrm{~Hz}-1.5 \mathrm{Khz}$ ) et moyennés (100 passages). La stimulation auditive est constituée soit de clicks, soit de bouffées tonales de différentes fréquences, générée par un stimulateur Nicolet SM 700, au travers d'écouteurs de type TDH 39. L'intensité de stimulation est de $70 \mathrm{~dB}$ au dessus du seuil auditif.

\section{3-RESULTATS}

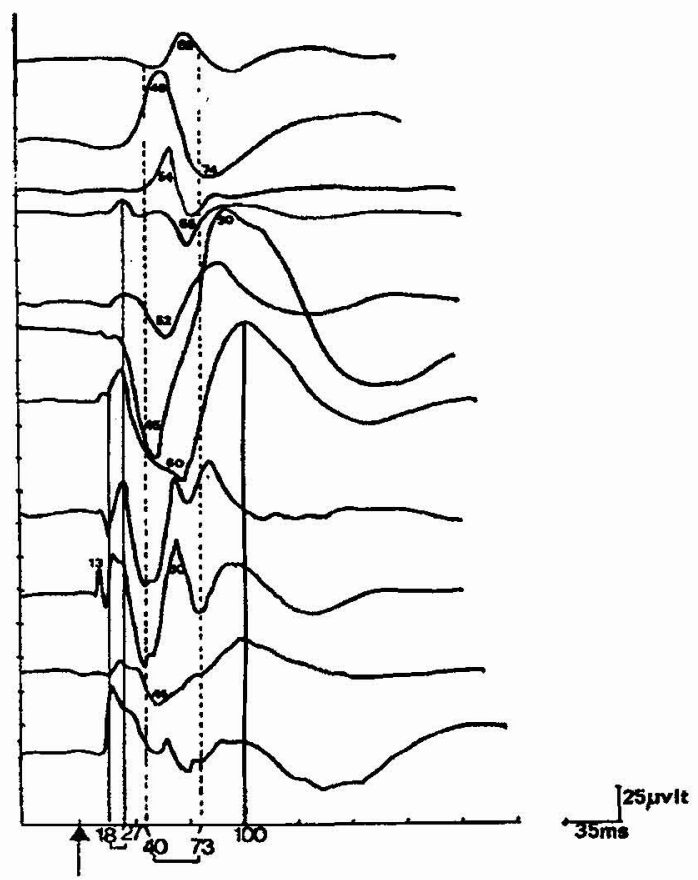

La Fig.2 montre les différentes composantes présentes dans un potentiel évoqué auditif intracérébral enregistré en réponse à une bouffée tonale. Chacune des courbes représente la réponse évoquée en un plot donné chez 11 patients différents. Nous pouvons séparer les composantes en trois catégories selon leurs latences. Nous distinguons 1-réponses à brève latence (13 à $17 \mathrm{msec}) ; 2$-à moyenne latence ( 40 à $75 \mathrm{msec}) ;$ et 3- à longue latence ( 100 msec et plus).

Fig 2. PEAs enregistrés en divers sites du Gyrus de Heschl (V. texte). La flèche désigne le départ de la stimulation auditive 


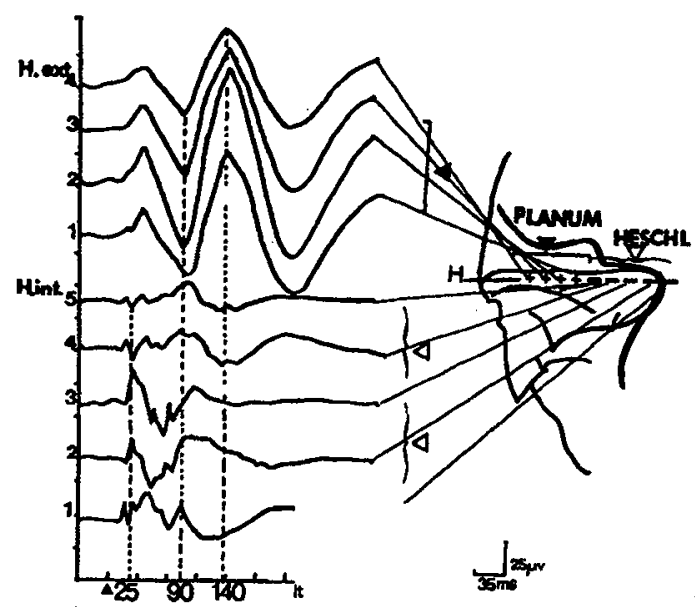

Fig.3 PEA enregistré en réponse d̀ une bouffée tonale sur chaque plot d'une même électrode

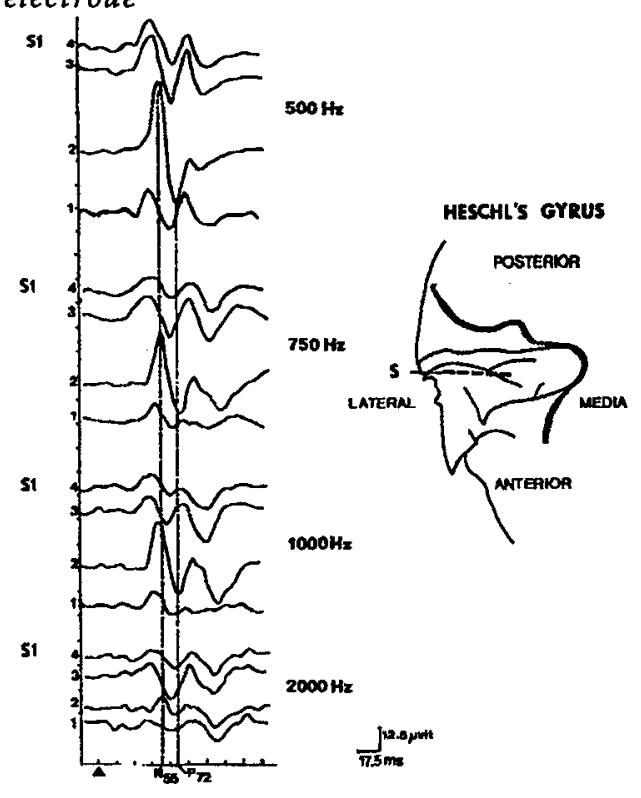

Il existe une ségrégation anatomique des réponses en fonction de leur latence. Ceci est illustré par la Fig.3. Les plots internes de l'électrode $\mathbf{H}$ (représentés par des pointillés) explorent la partie postero-dorso-médiane du gyrus de Heschl et les plots externes (croix) le planum temporale. Les réponses évoquées à une bouffée tonale le long de cette électrode sont représentées par les courbes à gauche de la figure. Les composantes a brève latence (13-25 $\mathrm{msec})$ sont enregistrées uniquement au niveau du gyrus de Heschl ( 5 premieres traces) et celle à longue latence $(140 \mathrm{msec}$ ) au niveau du Planum Temporale. Les réponses a moyennes latences $(40-75 \mathrm{msec})$ sont clairement identifiables au niveau du gyrus de Heschl mais peuvent être présentes également, de plus faible amplitude, au niveau du Planum Temporale.

Les réponses à brève latence présentent les caractéristiques de réponses primaires et ne varient pas (en latence, ni en amplitude) en fonction de la fréquence tonale. Par contre, l'amplitude des composantes a moyenne latence est fonction de la fréquence. La Fig.4. montre les variations d'amplitude des composantes N55-P72 Elles présentent un, maximum sur le plot $\mathrm{N}^{\circ} 2$ lorsque la bouffée tonale est de fréquence $500 \mathrm{~Hz}$ et diminuent au fur et à mesure que la fréquence appliquée devient plus aigüe $(2000 \mathrm{~Hz})$. L'électrode sur laquelle sont enregistrés ces PEA est située dans la partie dorso-latérale du gyrus de Heschl

\section{Fig 4. PEA obtenu en réponse à des bouffées tonales de différentes fréquences sur} chacun des plots d'une électrode

En appliquant cette méthode de mesure des maxima des composantes à moyennes latences en fonction des fréquences tonales en différents sites du gyrus de Heschl, nous avons pu observer qu'il existait une organisation tonotopique du cortex auditif. Les hautes fréquences sont représentées médialement et les basses fréquences latéralement. 


\section{DISCUSSION}

Nos résultats montrent que les potentiels évoqués enregistrés au niveau du cortex auditif chez des sujets éveillés sont composés de trois groupes de composantes séparées par leurs latences (Fig 2).Les composantes à courte latence sont comparables à celles précédemment décrites par Celesia et Puletti (1969) et corroborées par Lee et al (1984) enregistrées avec des électrodes subdurales. Elles présentent les caractéristiques de réponses primaires et sont préférentiellement obtenues dans la partie dorso-postéro-médiane du gyrus de Heschl (LiegeoisChauvel et col, 1989, soumis pour publication). Cette région constituerait l'aire primaire, décrite comme un koniocortex par les données cytoarchitectoniques de Galaburda et Sanides (1980). Mais il est peu propable, compte tenu de leur localisation, qu'elles puissent être enregistrées sur le scalp.

Les composantes à moyenne latence (CML) et celles à longue latence (CLL) ont des générateurs situés dans des aires différentes. Les réponses à moyenne latence sont enregistrées au niveau du gyrus de Heschl et celles a longue latence au niveau du Planum Temporale.(Fig 3) Ces résultats renforcent l'hypothèse selon laquelles les CML enregistrées sur le scalp peuvent être générées au niveau du cortex auditif (Wood et Wolpaw, 1982; Scherg et Von Cramon, 1986). Par contre, ils sont en opposition avec les données apportées par la magnétoencéphalographie (MEG) qui montrent que le PEA magnétique et que le PEA électrique d'une latence de $50 \mathrm{~ms}$ ont une même source, située au niveau du cortex du Planum Temporale (Reite et coll, 1988). La finesse de nos plots d'enregistrement et la précision de la reconstruction anatomique de leurs positions ( Musolino et col,1989) nous permettent d'affiner la localisation des générateurs de ces réponses.

L'amplitude de ces CML varie en fonction de la fréquence appliquée (Fig 4) et montre qu'il existe une distribution tonotopique des fréquences tonales au niveau du gyrus de Heschl. Les hautes fréquences seraient représentées médialement et les basses fréquences latéralement, organisation similaire à celle qui a été décrite chez le singe (Merzenich et Brugge, 1973)'et chez l'Homme en MEG par Romani et Williamson (1982). Il semble exister une carte tonotopique différente codée par les réponses à longue latence au niveau du Planum Temporale qui doit être confirmée par des données supplémentaires.

\section{REFERENCES}

1/Chatrian, G.E.,Petersen, M.C. and Lazarte, J.A.,EEG Clin Neurophysiol 12 (1959) 479.

2/Celesia, G. and Puletti, F., Neurology 19(1969) 211.

3/Szikla, G., Bouvier, G., Hori, T. et Petrov, V. Atlas of vascular patterns and stereotactic cortical localization. (1977) Springer Verlag

4/Musolino, A.,Talairach,J., Tournoux,P. et Missir, O., Boll. lega.It. Epil. 62/63 (1989) 51.

5/Lee,Y.S., Lueders, H., Dinner, R.P.,Lesser, R.P., Hahn,J. and Klem, G. , Brain 107 (1984) 115.

6/Liegeois-Chauvel,C.,, Musolino, A. et Chauvel,P (soumis pour publication ,Brain)

7/Galaburda,A. and Sanides, F., J. Comp.Neurol. 190 (1980) 597.

8/ Wood, C.C. et Wolpaw, J.R. , EEG Clin. Neurophysiol 54 (1982) 25.

9/Scherg,M; et Von Cramon, D. , EEG Clin. Neurophysiol 65 (1986) 344.

10/Reite, M., Teale,P., Zimmerman, J. Davis, K. et Whalen,J., EEG. Clin. Neurophysiol. 70 (1988)

490

11/Merzenich, M.M. et Brugge, J.F., Brain Res. 50 (1973 ) 275

12/Romani, G.L., Williamson, S.J. et Kaufman, L. , Science 216 (1982) 1339. 